\title{
HLA typing for DR3 and DR4 using artificial restriction fragment length polymorphism PCR from archival DNA
}

\author{
V A Horton, M Bunce, D R Davies, R C Turner, Y-M D Lo
}

\begin{abstract}
Aim-To develop polymerase chain reaction based artificial restriction fragment length polymorphism (artificial RFLP PCR) assays for DR3 and DR4 alleles of the multiallelic DRB1 locus and to apply them to paraffin wax embedded archival material.

Methods-Sixty five samples from DRB1 typed cell lines were analysed using the artificial RFLP PCR method to determine the specificity and sensitivity of the system.

Results-The artificial RFLP PCR method for typing the DRB1 locus showed $100 \%$ accuracy in the 65 samples previously typed using allele specific PCR and serology. The samples included 18 combinations of alleles that included DR3, 18 that included DR4, four that were DR3/DR4 heterozygotes, and 10 samples that were neither DR3 nor DR4. Typing of 10 paraffin wax embedded samples using artificial RFLP PCR was in complete agreement with previous typing at the DRB1 locus.

Conclusion-The application of artificial RFLP PCR for the analysis of multiallelic loci, such as those of the HLA system, in archival DNA samples has been achieved. Artificial RFLP PCR is a robust, easily implemented, non-isotopic system and may be useful for large retrospective studies.

(F Clin Pathol 1995;48:33-36)
\end{abstract}

Diabetes Research Laboratories, Radcliffe Infirmary, Woodstock Road, Oxford OX2 6HE V A Horton

R C Turne

Oxford Transplant Centre, Churchill Hospital, Oxford $M$ Bunce

Department of Cellular Pathology, John Radcliffe Hospital, Oxford D R Davies

Nuffield Department of Clinical Medicine Y-M D Lo

Correspondence to: Mrs V A Horton.

Accepted for publication 1 July 1994
Keywords: HLA typing, artificial RFLP PCR, diabetes.

The genetic risk of several autoimmune diseases, including insulin dependent diabetes mellitus (IDDM), is linked to the major histocompatibility complex (MHC) class II loci on chromosome 6p. In IDDM there is a particularly strong association with certain "susceptibility" alleles of the DRB1 gene." These "susceptibility" alleles belong to two of the 11 allelic subgroups into which the 59 known alleles of DRB1 are subdivided. ${ }^{2}$ DR3 and DR4, comprising three and 12 alleles, respectively, are the allelic subgroups most highly associated with IDDM, with DR3/DR4 heterozygotes being at particularly high risk. ${ }^{3}$ The risk presented by the DR4 alleles is partly caused by the DQB $1{ }^{\star} 0302$ allele, which is in linkage equilibrium with DR4. ${ }^{4}$ The risk associated with DR3 alleles may be either because of DR3 itself or from linkage disequilibrium with other as yet unknown alleles. Reliable identification of DR3 and DR4 alleles in susceptible populations is helpful in identifying subjects who may be at risk of developing IDDM and assists in determining the role of these alleles in the aetiology and pathogenesis of insulin dependent diabetes.

Typing of individuals at the DRB1 locus is complicated as there are 59 known alleles and at least three other loci in the region containing alleles or pseudogenes that have homology to DRB1 (fig 1). ${ }^{5}$ All of these variants are distinguishable only by a few base pair changes with or without amino acid changes in their hypervariable regions. Techniques for complete typing at this locus are often laborious, such as sequence specific oligonucleotide probing and restriction fragment length polymorphism (RFLP) analyses. ${ }^{67}$ Serological methods are dependent upon the availability of suitable reagents and can require repeated typing to obtain specificity. The current much used method is allele specific polymerase chain reaction (PCR), also called the amplification refractory mutation system (ARMS), which is much easier and faster than those mentioned above. ${ }^{8-10}$ However, ARMS can sometimes be unreliable when the quality and quantity of the material to be typed is different from the controls upon which the PCR was developed. ${ }^{11}$ ARMS PCR is therefore potentially unreliable when applied to DNA samples from a variety of sources-for example, archival material, because false negative results can occur when samples are of lower purity and not as concentrated as the control samples. Nested PCR has been used in conjunction with ARMS on degraded samples but this process involves multiple successive PCR reactions and requires tedious dilutions before the allele specific step. ${ }^{12}$

To overcome the difficulties associated with degraded and impure samples, we have developed a specific artificial RFLP PCR assay for DR3 and DR4. In artificial RFLP PCR a base change near the $3^{\prime}$ end of a primer is introduced to create a site recognised by a restriction enzyme. ${ }^{13}$ The site introduced should be specific for the particular allele or, as in this case, alleles of interest. In our method Sau 3A 1 and Spe 1 sites are created to identify DR4 and DR3 alleles, respectively. Sixty five samples from cell 
lines that were DRB1 typed using serological and allele specific methods were analysed using artificial RFLP PCR to determine specificity and sensitivity. The method was then applied to paraffin wax embedded archival material.
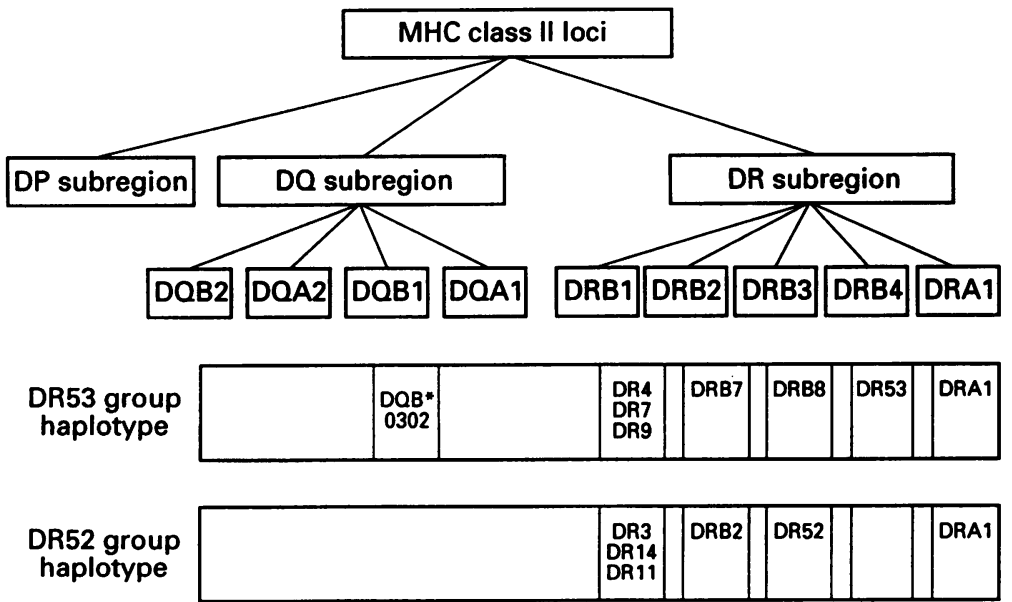

Figure 1 Schematic diagram of the genomic localisation of the MHC class II molecules and particularly the $D Q$ and $D R$ subregions, each of which include several loci. Two of the $D R$ loci, DRB2 and DRB3, contain alleles that are pseudogenes. Two common haplotypes, the DR53 group and the DR52 group, exhibit linkage disequilibrium which can occur between the DR3 and DR4 alleles at the DRB1 locus and alleles at other loci. Thus, the pseudogene $D R B 7$ is found with greater frequency than expected by chance with certain DRB1 alleles, including DR4.

A DR4 ${ }^{+}$allele
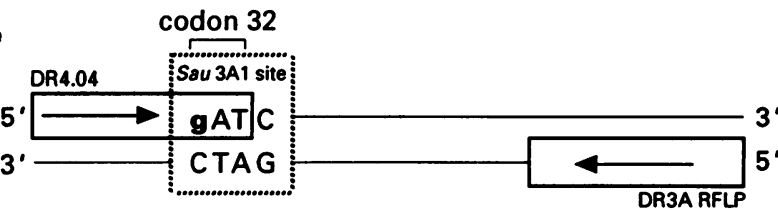

DR4- allele
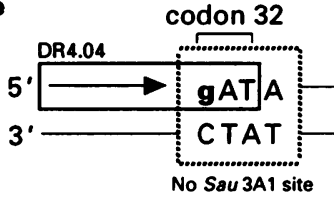

B $\mathrm{DR3}^{+}$allele

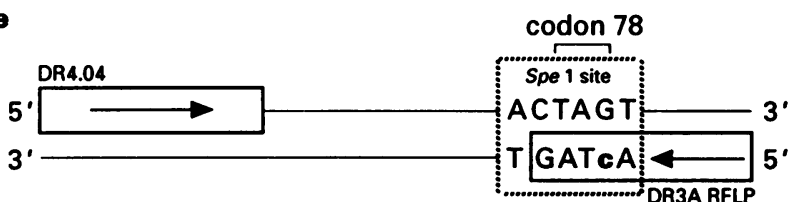

\section{DR3- allele}

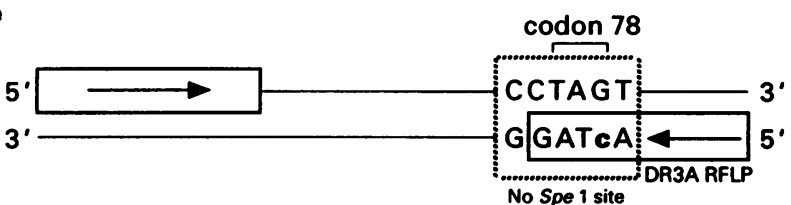

Figure 2 Diagrams illustrating the principles of artificial RFLP PCR for typing the DR4 and $D R 3$ alleles of the DRB1 gene. A:DR4 detection. $A$ deliberate mismatch of $C$ to $G$ at codon 32 is introduced in the $3^{\prime}$ end of the primer. Sau $3 A 1$ cuts the resulting sequence $G A T C$, which is only present in the DR4 PCR product. $B: D R 3$ detection. A deliberate mismatch of $G$ to $C$ at codon 78 is introduced in the 3 ' end of the primer. Spe 1 cuts the resulting sequence $A C T A G T$, which is only present in DR3 and in the DRB7 pseudogene PCR products.

\section{Methods}

Genomic DNA samples from 65 cell lines in this study were kindly provided by $\mathrm{Dr} \mathrm{K}$ Welsh of the Oxford Transplant Centre (Oxford, UK) and were previously DRB1 typed using either allele specific PCR or serology. Paraffin wax embedded samples were provided by Dr D Davies and were also previously DRB1 typed using allele specific PCR or serology.

Paraffin wax embedded donor kidney tissues were randomly chosen from material collected in 1992. Ten $5 \mu \mathrm{m}$ sections were cut from paraffin wax blocks and placed in a $1.5 \mathrm{ml}$ Eppendorf tube. Subsequent processing was as described previously. ${ }^{14}$ Briefly, each section was dewaxed in $1.0 \mathrm{ml}$ xylene, rotary mixed for 30 minutes, centrifuged for five minutes at $12000 \times g$, and the xylene pipetted off. This process was repeated. To ensure removal of the xylene, $1 \mathrm{ml} \mathrm{95 \%} \mathrm{ethanol} \mathrm{was} \mathrm{added,}$ mixed for 30 minutes, centrifuged at 12000 $\times g$, and most of the ethanol pipetted off. The pellet was further dried by centrifuging at $37^{\circ} \mathrm{C}$ using a vacuum pump for 10 minutes. Digestion buffer $(100 \mu \mathrm{l})(50 \mathrm{mM}$ TRIS, pH 8.5, $1 \mathrm{mM}$ EDTA, 0.5\% Tween 20 (Sigma, Poole, Dorset, UK) with $200 \mu \mathrm{g} / \mathrm{ml}$ proteinase $\mathrm{K}$ (Boehringer-Mannheim, Lewes, UK)) was added to each tube. The pellet was digested with proteinase $\mathrm{K}$ overnight at $37^{\circ} \mathrm{C}$. After digestion, the tube was spun briefly and heated at $94^{\circ} \mathrm{C}$ for 20 minutes to inactivate proteinase $\mathrm{K} ; 10 \mu \mathrm{l}$ was then used for the PCR assay.

The primer sequences amplified all DRB1 alleles: DR3 artificial RFLP: 5' CCG CTG CAC TGT GAA GCT CTC CAC AAC CCC GTA GTT GTG TCT GCA CTAG 3' (antisense). DR4.04: 5' CGG GTG CGG TTC CTG GAC AGA TAC TTC GAT 3' (sense).

For the PCR reaction, primers were chosen for their proximity to a region unique to the particular group of alleles to be typed and for the ability to create, on modification of the primer, part of a restriction site that will only be present upon amplification of those specific alleles. The partial restriction site is located at the 3' end of the primer and any base change, provided it is not located at the last base of the primer, will be incorporated into the product. Introducing a base change at the end of a primer makes it refractory to amplification. ${ }^{15}$ The sense primer was altered from the wild-type DR4 alleles and the antisense primer from wild-type DR3 alleles. Because the primers are in regions which are highly conserved among the various alleles, they are able to amplify all or at least most alleles tested. The base change in the sense primer produces a Sau 3A1 site on amplification of DR4 alleles (fig 2A). The base change in the antisense primer creates part of a $S p e 1$ site that, on amplification of a DR3 or a DRB7 allele, results in the formation of the full site (fig 2B). The DRB7 restriction fragments are the same size as those of DR3. A third enzyme, Dde 1, which recognises a CTNAG site in the DRB7 pseudogene not 
Figure $3 \quad P C R$ products from individuals with $D R 4, D R 3, D R 3 / 4$, $D R B 7$, a Dde 1 positive but non-DRB7 site

(DNS), and without any of the above $(N)$. The upper part of the gel shows $N, D R 4, D R B 7, D R B 7$, DNS, DNS, DR3/4, N, $N, D R 4$, and pBR322/Msp 1 size markers; the lower part of the gel shows $N, N, N, N$ $N, D R 4, N, D R 3 / D R 4$, $N, D R 3$, and PBR322/Msp 1 size markers.

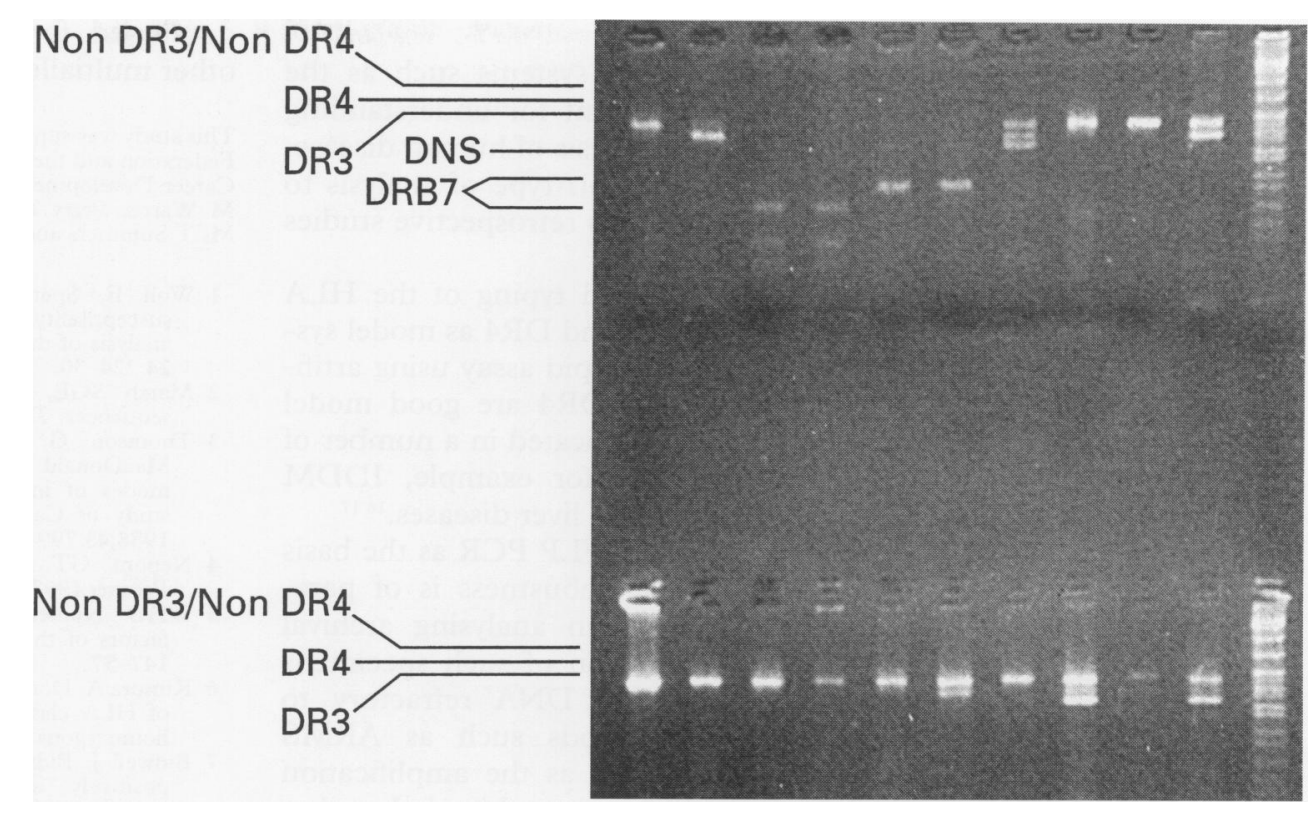

present in DR3 alleles, is used to digest DRB7, so that the assay can differentiate between alleles.

PCR was carried out in $50 \mu \mathrm{l}$ reaction volumes with primer concentrations at 50 pmoles/reaction. Two units of Taq polymerase (Promega, Madison, Wisconsin, USA) and 0.1 to $1.0 \mu \mathrm{g}$ of DNA were used. Nucleotide triphosphates were obtained from Perkin-Elmer (Warrington, UK); $\mathrm{MgCl}_{2}$ concentration was at $2.0 \mathrm{mM}$. Cycling profile was 35 cycles of denaturing at $95^{\circ} \mathrm{C}$ for $20 \mathrm{sec}-$ onds, annealing at $55^{\circ} \mathrm{C}$ for 20 seconds, and extension at $72^{\circ} \mathrm{C}$ for 30 seconds, performed on a Perkin Elmer Cetus 9600 thermal cycler. Following amplification, $8 \mu \mathrm{l}$ of sample was loaded onto a $1.5 \%$ agarose gel for visualisation by ultraviolet transillumination. An amplification product of 213 base pairs was generated.

PCR product $(12 \mu \mathrm{l})$ was digested first with 20 units each of Spe 1 (Boehringer Mannheim) and Dde 1 (New England Biolabs, Beverley, Massachusets, USA) in a volume of $15 \mu \mathrm{l}$ for two hours at $37^{\circ} \mathrm{C} ; 12 \mu \mathrm{l}$ of this reaction was then digested with 20 units of Sau 3A1 (New England Biolabs) in a volume of $15 \mu \mathrm{l}$ for two hours at $37^{\circ} \mathrm{C}$. The restriction fragments were then size-fractionated on a $3.5 \%$ metaphor agarose gel and run at 90 volts for one hour. DNA was visualised by ultraviolet transillumination after staining with ethidium bromide. The DR3 and DR4 alleles were recognised by the presence of 164 and 186 base pair fragments, respectively.

\section{Results}

The alleles obtained in patients with DR4, DR3, DRB7, and DRB4 are presented in fig 3.

The primers used in artificial RFLP PCR were designed to ensure amplification of all DRB1 alleles. Long primers and a relatively low annealing temperature were used to accommodate base pair differences among alleles in the primer binding region and amplified a 213 base pair DNA fragment. Digestion by Sau 3A1 of the GATC sequence that only occurs in DR4 alleles gave a diagnostic 186 base pair fragment (fig 2A). Digestion of the ACTAGT that only occurs in those with either DR3 or DRB7 alleles by Spe 1 gave a 164 base pair fragment (fig 2B). The restriction enzyme Dde 1 distinguished between the alleles because it recognised two CTNAG sites in DRB7, at codons 51-52 and 58-59, and none in DR3. On digestion of a DRB7 allele with Dde 1, the PCR product was cut into three fragments: 106,86 , and 21 base pairs, although the latter was too small to detect reliably. In samples positive for alleles containing a Dde 1 site other than the DRB7 allele the second cutting site was absent so that a $106 / 107$ base pair doublet was observed.

The artificial RFLP PCR method for typing the DRB1 locus showed $100 \%$ sensitivity and specificity in typing 50 samples previously typed using allele specific PCR and serology. The samples included 18 combinations of alleles that included DR3, 18 combinations that included DR4, four DR3/DR4 heterozyDR4. To assess the specificity of detecting DR3 from DRB7, 15 additional samples were chosen containing alleles known to be in linkage disequilibrium with DRB7. In one DRB7 was apparent by the specific fragments produced on digestion with $D d e 1$.

The method was then applied to paraffin wax embedded archival kidney tissue. The protocol was modified slightly to compensate for the quality of the DNA sample, with the cycle number increased to 40 to obtain sufficient product for analysis. Typing of 10 paraffin wax embedded kidney samples using artificial RFLP PCR was in complete agreement with the previous typing using allele specific PCR and serology. These included three DR4 positive and two DR3 positive gotes, and 10 samples with neither DR3 nor samples. 
Discussion

Analysis of multiallelic systems such as the HLA system is important for understanding the molecular pathogenesis of human disease. The ability to extend this type of analysis to archival material permits retrospective studies to be undertaken.

In this study we used typing of the HLA alleles coding for DR3 and DR4 as model systems for developing a rapid assay using artificial RFLP. DR3 and DR4 are good model systems as they are implicated in a number of autoimmune diseases-for example, IDDM and certain autoimmune liver diseases. ${ }^{16} 17$

We chose artificial RFLP PCR as the basis of our assay because robustness is of paramount importance when analysing archival materials as a proportion of such specimens may contain degraded DNA refractory to amplification. ${ }^{1819}$ Methods such as ARMS may not be satisfactory as the amplification and diagnostic steps are combined. In other words, a negative result may be ambiguous as it could potentially be caused by the absence of the tested allele or the absence of amplification because of sample degradation or the presence of PCR inhibitors. The use of control primers-that is, those that amplify a product that should be present in all samples, along with the ARMS primers can help ensure that a negative result is not caused by PCR inhibitors, but is not sufficient in all cases. This is because the control and ARMS reactions represent different PCRs and therefore may fail or succeed in amplifying the target independently of each other. In artificial RFLP analysis the amplification step is separated from the diagnostic phase involving the use of restriction enzymes. In patients with and without the allele under investigation amplified DNA is visualised so the identification of unamplifiable samples can be distinguished from a negative result.

Our data have demonstrated the applicability and robustness of artificial RFLP systems to situations where partially degraded DNA may be present. The system is easily implemented and is non-isotopic. An artificial RFLP system, similar to the one described in this paper, can be easily and rapidly developed for typing other HLA alleles or other multiallele systems.

This study was supported by grants from the Juvenile Diabetes Federation and the Wellcome Trust. Y-MD Lo is a Wellcome Career Development Fellow. We are grateful for advice from $M$ Warren-Perry and for assistance from $T$ Bown and to Ms T Summers and Ms I Samuel for typing the manuscript.

1 Wolf E, Spencer KM, Cudworth AG. The genetic susceptibility to type 1 (insulin-dependent) diabetes: analysis of the HLA-DR association. Diabetologia 1983 24:224-30.

2 Marsh SGE, Bodmer JG. HLA class II nucleotide sequences. Tissue Antigens 1992;40:229-43.

3 Thomson G, Robinson WP, Kuhner MK, Joe S, MacDonald MJ, Gottschall JL. Genetic heterogeneity, modes of inheritance, and risk estimates for a joint modes of inheritance, and risk estimates for a joint study of Caucasians

4 Nepom GT. Immunogenetics and IDDM. Diabetes Reviews 1993;1:93-103.

5 WHO Nomenclature Committee. Nomenclature for factors of the HLA System, 1991. Vox Sang 1992;63: 147-57.

6 Kimura A, Dong K-P, Harada H, Sasazuki T. DNA typing of HLA class II genes in B lymphoblastoid cell line homozygous for HLA. Tissue Antigens 1992;40:5-12.

7 Bidwell J, Bidwell E. A DNA-RFLP typing system that positively identifies serologically well-defined and ill-defined HI A DR and DQ alleles including DRw10. Transplantation 1988;45:640-6.

8 Lo Y-MD, Mehal WZ, Wordsworth BP, Chapman RW, Fleming KA, Bell JI. HLA typing by double ARMS. Fleming KA, Bell JI.

9 Olerup O, Zetterquist H. HLA-DR typing by PCR amplification with sequence-specific primers (PCR-SSP) Tissue Antigens 1992;39:225-35.

10 Bunce M, Taylor CJ, Welsh KI. Rapid HLA-DQB typing by eight PCR amplifications with sequence-specific primers (PCR-SSP). Hum Immunol 1993;37:201-6.

11 Lo ESF, Lo Y-MD, Tse CH, Fleming KA. Detection of a hepatitis $B$ pre-carc mutant by allele specific polymerase chain reaction. $\mathcal{f}$ Clin Pathol 1992;45:689-92.

12 Bein G, Glaser H, Kirchner. Rapid HLA-DRB1 genotyping by nested PCR amplification. Tissue Antigens 1992;39: b8 -73 .

13 Patel P, Lo Y-MD, Bell JI, Wainscoat JS. Detection of susceptibility alleles to IDDM at the DQB1 locus by artificial PCR-RFLP. Immunogenetics 1992:36:264-5.

14 Lo Y-MD, Lo E S-F, Mehal WZ, Sampietro M, Fiorelli $G$, Ronchi G. Geographical variation in prevalence of hepatitis B virus DNA in $\mathrm{HBsAg}$ negative patients. f Clin Pathol 1993;46:304-8.

15 Newton CR, Graham A, Heptinstall LE, Powell SJ, Summers C, Kaisheker N. Analysis of any point mutation in DNA. The amplification refractory mutation system (ARMS). Nucleic Acids Res 1989;17: 2503-16.

16 Chapman RW. Role of immune factors in the pathogenesis of primary sclerosing cholangitis. Semin Liver Dis 1991; of primary 1 . 4 .

17 Seki T, Kiyosawa $K$, Inoko $H$, Ota $M$. Association of autoimmune hepatitis with HLA-Bw54 and DR4 in Japanese patients. Hepatology 1990;12:1300-4.

18 Lo Y-MD, Mehal WZ, Fleming KA. In vitro amplification of hepatitis $B$ virus sequences from liver tumour DNA and from paraffin wax embedded tissues using the polymerase chain reaction. $\mathcal{f}$ Clin Pathol 1989;42:840-6.

19 An SF, Franklin D, Fleming KA. Generation of digoxigenin-labelled double-stranded and single-stranded probes using the polymerase chain reaction. Mol Cell Probes 1992;6:193-200. 